\title{
REVIEWS
}

\section{The Impact of Social Determinants of Health on Medication Adherence: a Systematic Review and Meta-analysis}

\author{
Marcee E. Wilder, M.D., M.P.H. ${ }^{7}$ (D) Paige Kulie, M.P.H. ${ }^{7}$, Caroline Jensen, M.D. ${ }^{2}$, \\ Paul Levett, M.Sc., MA. Ed. ${ }^{3}$, Janice Blanchard, M.D., Ph.D., M.P.H. ${ }^{\text {, }}$, \\ Luis W. Dominguez, M.D., M.P.H. ${ }^{\text {, }}$, Maria Portela, M.D., M.P.H. ${ }^{\text {, }}$, \\ Aneil Srivastava, B.S., B.A. ${ }^{7}$, Yixuan Li, Ph. D. ${ }^{4}$, and Melissa L. McCarthy, Sc.D. ${ }^{1,4}$
}

\begin{abstract}
'Department of Emergency Medicine, George Washington University, Medical Faculty Associates, Washington, DC, USA; ${ }^{2}$ Anesthesiology Residency Program, Columbia University, New York, NY, USA; ${ }^{3}$ Himmelfarb Health Sciences Library, George Washington University, Washington, DC, USA; ${ }^{4}$ Department of Health Policy and Management, Milken Institute School of Public Health, George Washington University, Washington, DC, USA.
\end{abstract}

BACKGROUND: Medication adherence (MA) is critical to successful chronic disease management. It is not clear how social determinants of health (SDH) impact MA. We conducted a systematic review and meta-analysis to summarize the evidence on the relationship between SDH and MA.

METHODS: We conducted a systematic review of the literature using a Preferred Reporting Items for Systematic Reviews and Meta-Analysis (PRISMA) format. A literature search was performed using three databases: PubMed, Scopus, and Cochrane Clinical Trials Register in December of 2018. Included studies were completed in the USA, included adults aged 18 years and older, measured at least one social determinant of health, and medication adherence was the primary outcome measure. Data from included full texts were independently extracted using a standardized data extraction form. We then conducted a meta-analysis and pooled the odds ratios from the included studies for each social determinant as well as for all SDH factors collectively.

RESULTS: A total of 3137 unduplicated abstracts were identified from our database searches. A total of 173 were selected for full text review after evaluating the abstract. A total of 29 articles were included for this systematic review. Economic-related SDH factors and MA were mostly commonly examined. The meta-analysis revealed a significant relationship between food insecurity $(\mathrm{aOR}=0.56$; 95\% CI 0.42-0.7), housing instability (aOR = 0.64; 95\% CI $0.44-0.93$ ), and social determinants overall (aOR $=0.75$; 95\% CI 0.65-0.88) and medication adherence.

DISCUSSION: Food insecurity and housing instability most consistently impacted medication adherence. Although included studies were heterogenous and varied widely in SDH and MA measurements, adverse social determinants overall were significantly associated with

This study was registered with the International Prospective Register of Systematic Reviews. Registration ID CRD42019119105

Prior Presentations: None.

Supplementary Information The online version contains supplementary material available at https://doi.org/10.1007/s11606-020-06447-0.

Received May 15, 2020

Accepted December 13, 2020

Published online January 29, 2021 lower MA. The relationship between SDH and MA warrants more attention and research by health care providers and policymakers.

KEY WORDS: social determinants of health; medication adherence.

J Gen Intern Med 36(5):1359-70

DOI: $10.1007 /$ s11606-020-06447-0

(C) Society of General Internal Medicine 2021

\section{INTRODUCTION}

As the prevalence of chronic conditions continues to increase in developed countries including the United States (US), adherence to long-term therapies is critical. One out of every two adults in the US have a chronic condition and $26 \%$ have two or more conditions. ${ }^{1}$ Chronic conditions are the main cause of poor health, disability, and death and account for the majority of health care costs. Many medications exist to manage chronic illnesses; however, their effectiveness is substantially reduced because half of all patients do not take their medication(s) as prescribed. ${ }^{1}$ Addressing and closing the medication adherence gap would significantly improve the ability of biomedical advances to reduce the burden and costs associated with chronic illness. ${ }^{2}$

The World Health Organization posits that medication adherence is influenced by patient, health care team/health system, therapy, disease condition, and socioeconomic-related factors. ${ }^{3}$ The majority of research to date has focused on patient-related factors associated with nonadherence such as forgetting doses, lifestyle barriers, and inadequate selfmanagement skills. ${ }^{3}$ Less attention has been paid to the other factors, particularly socioeconomic-related barriers or social determinants of health (SDH), with the exception of health insurance coverage. There is strong evidence of the negative consequences of the lack of health insurance coverage on medication adherence. ${ }^{4-6}$

Understanding the influence of SDH on medication adherence is important. Adverse social and living circumstances not only contribute to a higher prevalence of chronic disease, but 
these same factors influence people's abilities to manage their health problems. It is plausible that one of the underlying mechanisms by which adverse social determinants impact health care use and health is through medication adherence. When people are struggling with housing instability, food insecurity, and/or unemployment, they likely do not have the necessary emotional and material resources to devote adequate attention to their health and treatment regimen.

The purpose of this systematic review is to examine the evidence of SDH factors, other than health insurance coverage, on MA. Although there are published systematic reviews in the literature on this topic, they have all focused on the relationship between $\mathrm{SDH}$ and MA for one disease condition such as HIV/AIDS, and usually evaluated the influence of only one SDH factor. ${ }^{7-12}$ For example, Singer et al. conducted a systematic review of studies that examined the relationship between food insecurity and adherence to antiretroviral therapy. ${ }^{12}$ The aim of this systematic review and meta-analysis is to summarize the evidence of the relationship between social determinants of health and medication adherence for a wide variety of SDH factors and chronic disease conditions since there is no evidence to suggest that social determinants significantly impact medication adherence in one disease process over another.

\section{METHODS}

Search Methods. We followed the Preferred Reporting Items for Systematic Reviews and Meta-Analysis (PRISMA) format ${ }^{13}$ and registered our systematic review protocol with PROSPERO, an international database of prospectively registered systematic reviews in health and social care. ${ }^{13,14}$ The objective of our search strategy was to identify all studies that examined the influence of one or more social determinant of health on medication adherence. To identify SDH factors, we used the Healthy People 2020 (HP2020) SDH model which includes five dimensions: (1) economic stability; (2) education; (3) social and community context; (4) health and health care; and (5) neighborhood and the built environment. ${ }^{15}$ The lead author worked with an information specialist, a co-author of this paper, to identify the relevant $\mathrm{MeSH}$ terms and keywords for each of the five SDH dimensions and subdomains as well as our main outcome, MA. Once the search terms were identified, we developed our search strategy and then searched PubMed, Scopus, and Cochrane Clinical Trials Register in December of 2018 for potentially eligible studies. A full description of the search terms and strategy we employed is shown in Table 1 . We did not perform a supplementary resource hand-search.
Study Selection. We used the following inclusion criteria to determine study eligibility: (a) the study was conducted in the United States; (b) the study participants were adults aged 18 years and older; (c) the study measured at least one SDH as determined by HP2020; (d) the study evaluated MA as the primary outcome measure; (e) the study was published within the past 25 years in a peer-reviewed journal as the results of original research; and (f) the study was written in English. As previously mentioned, studies were excluded if they focused solely on the relationship between health insurance coverage and MA or cost-related MA. We limited included studies to the United States as there are large differences in social support programs and per capita spending on social services which affects the health outcomes of other developed nations. ${ }^{16}$

We imported all references into RefWorks, and the lead author deleted duplicates. The lead author divided the references among three reviewers who conducted an initial screen to determine study eligibility based on a review of each study's title and abstract. Prior to completing the initial screen, the lead author trained the other two reviewers on the study eligibility criteria and conducted a reliability assessment to determine whether there was good agreement among the three reviewers regarding whether a study met the eligibility criteria.

To assess the interrater reliability of study eligibility, we selected a random sample of 50 abstracts and each of the three reviewers independently read the title and abstract and determined study eligibility. The overall agreement between the three reviewers was $90 \%$ and the kappa statistics were 0.85 and 0.90 . There were five cases of disagreement, these cases were discussed, and then, each reviewer proceeded to independently screen one-third of the total abstracts for study eligibility. The three reviewers discussed any questions related to study inclusion or exclusion with each other and the senior author as needed. The three reviewers either flagged an abstract for full text review or documented the reason(s) for exclusion.

Data Extraction. Due to the large number of abstracts identified for full text review, we asked three additional reviewers to participate in the full text review. The first author trained all reviewers on study eligibility criteria and data extraction. Six reviewers read and evaluated one-sixth of the included full texts to determine whether the studies met the eligibility criteria and, if not, detailed the reasons for exclusion. For those articles that met the eligibility criteria, the reviewers used a standardized data form developed by the study team to independently extract the following data elements from each article: name of first author, year of publication, journal title, location, social determinant(s) of health examined, medication adherence measures, study population including disease/illness, how the study cohort was selected, sample size, purpose of the study, main results and statistical significance if applicable. 
Table 1 Search Terms and Abstracts Identified for Further Screening

\begin{tabular}{|c|c|c|}
\hline & Search topic & Search terms \\
\hline \multirow{7}{*}{ Economic Stability } & Medication adherence & $\begin{array}{l}\text { (medication OR prescription OR prescribe) AND (adherence OR compliance OR } \\
\text { obedience OR consent) AND }\end{array}$ \\
\hline & United States & United States AND \\
\hline & Employment & "Employment"[Mesh] OR \\
\hline & Food insecurity & "Food Supply”[Mesh] OR “Food Insecurity” OR \\
\hline & Housing instability & (“Housing”[Mesh] OR “Public Housing”[Mesh] OR “Homeless Persons"[Mesh]) OR \\
\hline & Poverty & "Poverty"[Mesh] OR \\
\hline & Income & Income [Mesh] OR \\
\hline \multirow[t]{4}{*}{ Education } & $\begin{array}{l}\text { Early childhood education and } \\
\text { development }\end{array}$ & $\begin{array}{l}\text { “Child, Preschool”[Mesh] AND (“Learning”[Mesh] OR "Education”'[Mesh] OR } \\
\text { "Educational Status"[Mesh] OR “Child Development”[Mesh] OR "Developmental } \\
\text { Disabilities"[Mesh]) OR }\end{array}$ \\
\hline & Enrollment in higher education & $\begin{array}{l}\text { ("Students”[Mesh] OR “Universities”'[Mesh] OR “Education, Graduate”[Mesh] OR } \\
\text { "Higher Education”[All]) OR }\end{array}$ \\
\hline & High school graduation & (“Schools”[Mesh] OR “"High School”[All]) OR \\
\hline & Language and literacy & ("Language"[Mesh] OR "Literacy"[Mesh]) OR \\
\hline \multirow{6}{*}{$\begin{array}{l}\text { Social and community } \\
\text { context }\end{array}$} & Civic participation & "Community Participation"[Mesh] OR \\
\hline & Discrimination & $\begin{array}{l}\text { ("Health Status Disparities"[Mesh] OR "Healthcare Disparities"[Mesh] OR "Minority } \\
\text { Health"[Mesh] OR "Social Discrimination"[Mesh] OR "Vulnerable }\end{array}$ \\
\hline & Incarceration & $\begin{array}{l}\text { Populations" }[\text { Mesh]) OR } \\
\text { ("Prisoners"[Mesh] OR "Prisons"[Mesh]) OR }\end{array}$ \\
\hline & Social cohesion & ("Socioeconomic Factors"[Mesh] OR "Social environment"[Mesh OR "Social \\
\hline & & Support”[Mesh] OR "Social Control, Formal”[Mesh] OR "Social \\
\hline & & Responsibility”[Mesh] OR “Social justice”[Mesh]) OR \\
\hline \multirow[t]{4}{*}{ Health and health care } & Access to health care & $\begin{array}{l}\text { (“Health Services Accessibility”[Mesh] OR “Medically underserved area”[Mesh]) } \\
\text { OR }\end{array}$ \\
\hline & Access to primary care & $\begin{array}{l}\text { ("Primary Health Care"[Mesh] OR "Health Services Accessibility”[Mesh] OR } \\
\text { "Health Services Needs and Demands”[Mesh]) OR }\end{array}$ \\
\hline & Health literacy & $\begin{array}{l}\text { "'Primary Health Care"'[Mesh] OR "Health Services Accessibility"[Mesh] OR } \\
\text { "Health Services Needs and Demands"[Mesh]) OR }\end{array}$ \\
\hline & Health & “Social Determinants of Health"[Mesh] OR \\
\hline \multirow{6}{*}{$\begin{array}{l}\text { Neighborhood and built } \\
\text { environment }\end{array}$} & Access to foods that support & (“Food Assistance”[Mesh] OR “Food Supply”[Mesh] OR “Fast Foods”[Mesh] OR \\
\hline & healthy eating patterns & "Diet, Food, and Nutrition"[Mesh] OR "Nutrition Policy”[Mesh]) OR \\
\hline & Crime and violence & $\begin{array}{l}\text { ("Crime"[Mesh] OR "Crime victims"[Mesh] OR "Violence"[Mesh] OR "Exposure to } \\
\text { violence"[Mesh] OR "Domestic violence"[Mesh] OR "Physical abuse"[Mesh]) OR }\end{array}$ \\
\hline & Environmental conditions & "Residence Characteristics"'[Mesh] \\
\hline & Quality of housing & (“Housing”[Mesh] OR “Public Housing”[Mesh] OR “Quality of Life”[Mesh]) \\
\hline & Built environment & Environment Design [Mesh] \\
\hline
\end{tabular}

For all studies that met the inclusion criteria, the senior author independently reviewed the results of each full text review to confirm eligibility, study methodological details, and main results. If there was any disagreement between the reviewer and the senior author regarding study eligibility, methodological details, or study results, the discrepancies were discussed with the lead author who served as the final arbitrator.

Data Synthesis and Analysis. To estimate the influence of SDH on medication adherence, we conducted a meta-analysis and pooled the odds ratios from the included studies for each $\mathrm{SDH}$ factor as well as overall. Some studies did not control for covariates and only reported the unadjusted odds ratio so we separately estimated a pooled adjusted and pooled unadjusted odds ratio (and respective 95\% confidence interval) for each SDH factor and overall. When needed, we inverted the odds ratio so that when we pooled the results we consistently estimated the adverse effect of a SDH factor on medication adherence. We were not able to include all of the studies in the meta-analysis because some studies measured medication adherence as a continuous outcome and there was not enough information provided in the published data to calculate an odds ratio (see Table 2).

We assessed the heterogeneity of the studies using Cochran's $Q\left(x^{2}\right.$ test) and $I^{2}$ test statistics and we estimated both the unadjusted and adjusted pooled odds ratios using random effects models ( $\mathrm{R}$ studio version 1.3.959: $\mathrm{R}$ package: metafor) because of the methodological differences among the studies.

\section{RESULTS}

Our literature review search yielded 3137 potential studies (see Fig. 1). After we removed the duplicates, we reviewed the title and abstract of 2583 citations and identified 173 articles for potential inclusion. After full text review, 29 of the 173 were included in this systematic review. The most common reasons for exclusion when both screening the abstracts and reading the full text articles were that the study did not include an SDH factor and/or the primary outcome was not medication adherence. 
Table 2 Characteristics of Included Studies

\begin{tabular}{|c|c|c|c|c|c|c|c|}
\hline $\begin{array}{l}\text { First } \\
\text { author, } \\
\text { name }\end{array}$ & Participants & $\begin{array}{l}\text { Study } \\
\text { design }\end{array}$ & $\begin{array}{l}\text { Medication } \\
\text { adherence } \\
\text { measure }\end{array}$ & $\begin{array}{l}\text { Social } \\
\text { determinant/ } \\
\text { s measured }\end{array}$ & Covariates & Main outcome & $\begin{array}{l}\text { Included } \\
\text { in pooled } \\
\text { analysis? }\end{array}$ \\
\hline $\begin{array}{l}\text { Bandi, } \\
2017^{17}\end{array}$ & $\begin{array}{l}1043 \text { low- } \\
\text { income His- } \\
\text { panic adults } \\
\text { with hyperten- } \\
\text { sion in NYC }\end{array}$ & $\begin{array}{l}\text { Cross- } \\
\text { sectional } \\
\text { survey }\end{array}$ & $\begin{array}{l}\text { Morisky } \\
\text { medication } \\
\text { adherence } \\
\text { scale (self- } \\
\text { report) }\end{array}$ & $\begin{array}{l}\text { Education, } \\
\text { employment }\end{array}$ & $\begin{array}{l}\text { Insurance status, } \\
\text { sex, marriage } \\
\text { status, age }\end{array}$ & $\begin{array}{l}\text { High school diploma } \\
\text { was associated with } \\
\text { hypertension adherence } \\
\text { among older Hispanics } \\
\text { compared to } \leq 8 \text { th } \\
\text { grade education but not } \\
\text { among younger } \\
\text { Hispanics }\end{array}$ & $\begin{array}{l}\text { Not } \\
\text { included } \\
\text { aPR = } 1.51 \\
1.06-2.14 \\
\text { vs aPR = } \\
1.06 ; \\
0.66-1.69\end{array}$ \\
\hline $\begin{array}{l}\text { Bauer, } \\
2013^{18}\end{array}$ & $\begin{array}{l}1336 \text { adults } \\
\text { with diabetes } \\
\text { and a new } \\
\text { antidepressant } \\
\text { prescription in } \\
\text { Northern, CA }\end{array}$ & $\begin{array}{l}\text { Prospective } \\
\text { cohort study }\end{array}$ & $\begin{array}{l}\text { New } \\
\text { antidepressant } \\
\text { prescription } \\
\text { medication } \\
\text { gap (claims- } \\
\text { based) }\end{array}$ & $\begin{array}{l}\text { Health } \\
\text { literacy, } \\
\text { income }\end{array}$ & $\begin{array}{l}\text { Demographics } \\
\text { including age, sex } \\
\text { and gender, } \\
\text { English } \\
\text { proficiency, family } \\
\text { size, education }\end{array}$ & $\begin{array}{l}\text { Low income associated } \\
\text { with more days with a } \\
\text { new prescription } \\
\text { medication gap than } \\
\text { higher income, literacy } \\
\text { limitations associated } \\
\text { with more days without } \\
\text { medications available } \\
\text { over the entire year }\end{array}$ & $\begin{array}{l}\text { Not } \\
\text { included } \\
\text { Income: }(\beta \\
=0.05 ; \\
0.00,0.09) \\
\text { Literacy: }(\beta \\
=0.06, p< \\
0.01)\end{array}$ \\
\hline $\begin{array}{l}\text { Billimek, } \\
2012^{19}\end{array}$ & $\begin{array}{l}3401 \text { diabetic } \\
\text { patients } \\
\text { receiving } \\
\text { medical care, } \\
\text { California }\end{array}$ & $\begin{array}{l}\text { Cross- } \\
\text { sectional } \\
\text { state survey }\end{array}$ & $\begin{array}{l}\text { Delay in filling } \\
\text { prescription } \\
\text { within past } \\
\text { year (self- } \\
\text { report) }\end{array}$ & $\begin{array}{l}\text { Food } \\
\text { insecurity }\end{array}$ & $\begin{array}{l}\text { Demographics, } \\
\text { income, education, } \\
\text { insurance, } \\
\text { psychological } \\
\text { distress, health } \\
\text { status }\end{array}$ & $\begin{array}{l}\text { Food insecurity } \\
\text { associated with delay in } \\
\text { filling prescription }\end{array}$ & Included \\
\hline $\begin{array}{l}\text { Bolkan, } \\
2013^{20}\end{array}$ & $\begin{array}{l}611 \text { veterans in } \\
\text { primary care } \\
\text { who screened } \\
\text { positive for } \\
\text { depression } \\
\text { across the US }\end{array}$ & $\begin{array}{l}\text { Secondary } \\
\text { analysis, } \\
\text { RCT }\end{array}$ & $\begin{array}{l}3 \text { questions } \\
\text { about taking } \\
\text { medication as } \\
\text { prescribed, } \\
\text { missing dose, } \\
\text { stopped taking } \\
\text { (self-report) }\end{array}$ & Social support & $\begin{array}{l}\text { Family } \\
\text { involvement }\end{array}$ & $\begin{array}{l}\text { Emotional and tangible } \\
\text { support were not } \\
\text { associated with } \\
\text { medication } \\
\text { nonadherence among } \\
\text { veterans receiving } \\
\text { primary care }\end{array}$ & Included \\
\hline Fan, $2016^{21}$ & $\begin{array}{l}208 \text { patients } \\
\text { from primary } \\
\text { care clinic with } \\
\text { type } 2 \text { diabetes } \\
\text { in St. Louis, } \\
\text { MO }\end{array}$ & $\begin{array}{l}\text { Cross- } \\
\text { sectional } \\
\text { survey }\end{array}$ & $\begin{array}{l}\text { Morisky } \\
\text { medication } \\
\text { adherence } \\
\text { scale (self- } \\
\text { report) }\end{array}$ & $\begin{array}{l}\text { Health } \\
\text { literacy, } \\
\text { income, } \\
\text { education }\end{array}$ & $\begin{array}{l}\text { Age, gender, race, } \\
\text { insurance, } \\
\text { medication } \\
\text { regimen } \\
\text { complexity, and } \\
\text { depression }\end{array}$ & $\begin{array}{l}\text { Health literacy was } \\
\text { associated with } \\
\text { unintentional adherence } \\
\text { but not associated with } \\
\text { overall adherence or } \\
\text { intentional } \\
\text { nonadherence }\end{array}$ & Included \\
\hline $\begin{array}{l}\text { Gerlach, } \\
2017^{22}\end{array}$ & $\begin{array}{l}458 \text { older } \\
\text { adults }(>60) \\
\text { with } \\
\text { depression } \\
\text { given new } \\
\text { antidepressant } \\
\text { prescription by } \\
\text { a physician in } \\
\text { Michigan }\end{array}$ & $\begin{array}{l}\text { Secondary } \\
\text { analysis of } \\
2 \\
\text { prospective } \\
\text { cohort } \\
\text { studies }\end{array}$ & $\begin{array}{l}\text { Single } \\
\text { question about } \\
\text { how } \\
\text { consistently } \\
\text { subject took } \\
\text { medication in } \\
\text { past week }\end{array}$ & Social support & $\begin{array}{l}\text { Age, education, } \\
\text { marital status, } \\
\text { illness burden, } \\
\text { cognitive function, } \\
\text { treatment site, } \\
\text { veteran status }\end{array}$ & $\begin{array}{l}\text { Among those with } \\
\text { inadequate social } \\
\text { support, antidepressant } \\
\text { medication adherence } \\
\text { was lower among older } \\
\text { blacks but not older } \\
\text { whites }\end{array}$ & Included \\
\hline $\begin{array}{l}\text { Johnson, } \\
2010^{23}\end{array}$ & $\begin{array}{l}275 \text { pharmacy } \\
\text { patients in } \\
\text { Atlanta, GA }\end{array}$ & $\begin{array}{l}\text { Cross- } \\
\text { sectional } \\
\text { survey }\end{array}$ & $\begin{array}{l}\text { Morisky } \\
\text { medication } \\
\text { adherence } \\
\text { scale (self- } \\
\text { report) }\end{array}$ & $\begin{array}{l}\text { Social } \\
\text { support, } \\
\text { health literacy }\end{array}$ & Age, sex & $\begin{array}{l}\text { There was a significant } \\
\text { interaction effect } \\
\text { between health literacy } \\
\text { and social support on } \\
\text { medication adherence }\end{array}$ & $\begin{array}{l}\text { Not } \\
\text { included }(\beta \\
=0.086 \\
0.018-0 \\
154)\end{array}$ \\
\hline $\begin{array}{l}\text { Kalichman, } \\
2010^{24}\end{array}$ & $\begin{array}{l}188 \text { HIV+ } \\
\text { adults taking } \\
\text { ART with low } \\
\text { health literacy, } \\
\text { Atlanta, GA }\end{array}$ & $\begin{array}{l}\text { Prospective } \\
\text { cohort }\end{array}$ & $\begin{array}{l}\text { Percent of pills } \\
\text { taken of those } \\
\text { dispensed in } \\
30 \text { days (self- } \\
\text { report) }\end{array}$ & $\begin{array}{l}\text { Food } \\
\text { insecurity and } \\
\text { housing } \\
\text { instability } \\
\text { summary } \\
\text { measure }\end{array}$ & $\begin{array}{l}\text { Social stress, AIDS } \\
\text { stigma, depression, } \\
\text { drug use }\end{array}$ & $\begin{array}{l}\text { Poorer ART adherence } \\
\text { significantly related to } \\
\text { nearly every indicator } \\
\text { of food insecurity }\end{array}$ & Included \\
\hline $\begin{array}{l}\text { Kalichman, } \\
2014^{25}\end{array}$ & $\begin{array}{l}344 \text { HIV+ } \\
\text { adults taking } \\
\text { ART, Atlanta, } \\
\text { GA }\end{array}$ & $\begin{array}{l}\text { Prospective } \\
\text { cohort }\end{array}$ & $\begin{array}{l}\text { Percent of pills } \\
\text { taken of those } \\
\text { dispensed } \\
\text { within 2-3- } \\
\text { month period } \\
\text { (self-report) }\end{array}$ & $\begin{array}{l}\text { Food } \\
\text { insecurity, } \\
\text { education, } \\
\text { income, } \\
\text { employment, } \\
\text { housing, } \\
\text { social support }\end{array}$ & $\begin{array}{l}\text { Depression, } \\
\text { alcohol use, drug } \\
\text { use }\end{array}$ & $\begin{array}{l}\text { Food insecurity was } \\
\text { protective against poor } \\
\text { adherence (i.e., }<80 \% \\
\text { adherent) }\end{array}$ & Included \\
\hline $\begin{array}{l}\text { Kripalani, } \\
2015^{26}\end{array}$ & $\begin{array}{l}1967 \text { patients } \\
\text { hospitalized } \\
\text { with acute } \\
\text { coronary } \\
\text { syndromes or } \\
\text { heart failure in } \\
\text { Nashville and } \\
\text { Franklin, TN }\end{array}$ & $\begin{array}{l}\text { Secondary } \\
\text { analysis- } \\
\text { Prospective } \\
\text { cohort study }\end{array}$ & $\begin{array}{l}\text { Adherence to } \\
\text { Refills and } \\
\text { Medications } \\
\text { Scale (self- } \\
\text { report) }\end{array}$ & $\begin{array}{l}\text { Health } \\
\text { literacy, } \\
\text { numeracy, } \\
\text { income, } \\
\text { education, } \\
\text { social support }\end{array}$ & $\begin{array}{l}\text { Health } \\
\text { competence, } \\
\text { depression }\end{array}$ & $\begin{array}{l}\text { 4-point change in health } \\
\text { literacy-increase in } \\
\text { the mean MA score } \\
\text { 2-point change in } \\
\text { numeracy-increase in } \\
\text { the mean MA } \\
\text { Income not associated } \\
\text { with MA }\end{array}$ & $\begin{array}{l}\text { Not } \\
\text { included } \\
\text { Health } \\
\text { literacy: }(\beta \\
=0.18 \\
0.02 \\
0.342)\end{array}$ \\
\hline
\end{tabular}


Table 2. (continued)

\begin{tabular}{|c|c|c|c|c|c|c|c|}
\hline $\begin{array}{l}\text { First } \\
\text { author, } \\
\text { name }\end{array}$ & Participants & $\begin{array}{l}\text { Study } \\
\text { design }\end{array}$ & $\begin{array}{l}\text { Medication } \\
\text { adherence } \\
\text { measure }\end{array}$ & $\begin{array}{l}\text { Social } \\
\text { determinant/ } \\
\text { s measured }\end{array}$ & Covariates & Main outcome & $\begin{array}{l}\text { Included } \\
\text { in pooled } \\
\text { analysis? }\end{array}$ \\
\hline & & & & & & $\begin{array}{l}\text { Education-significant } \\
\text { nonlinear association } \\
\text { with MA } \\
\text { Social } \\
\text { support-significant } \\
\text { nonlinear relationship } \\
\text { with MA }\end{array}$ & $\begin{array}{l}\text { Numeracy: } \\
(\beta=0.28 ; \\
0.10,0.46) \\
\text { Income: }(\beta \\
=0.06,- \\
0.12,0.24) \\
\text { Education: } \\
p<0.05 \\
\text { Social } \\
\text { support: } p \\
<0.05\end{array}$ \\
\hline $\begin{array}{l}\text { Markowitz, } \\
2011^{27}\end{array}$ & $\begin{array}{l}119 \text { adults with } \\
\text { HIV receiving } \\
\text { substance use } \\
\text { treatment in } \\
\text { Boston, MA }\end{array}$ & $\begin{array}{l}\text { Secondary } \\
\text { analysis- } \\
\text { RCT }\end{array}$ & $\begin{array}{l}\text { Percent of } \\
\text { adherence in } \\
\text { past } 7 \text { days } \\
\text { (electronic pill } \\
\text { cap system) }\end{array}$ & $\begin{array}{l}\text { Violence: } \\
\text { child sexual } \\
\text { abuse }\end{array}$ & $\begin{array}{l}\text { Age, years of } \\
\text { education, gender, } \\
\text { and race }\end{array}$ & $\begin{array}{l}\text { Child sexual abuse } \\
\text { prior to } 13 \text { years old or } \\
\text { between ages of } 13 \text { and } \\
16 \text { was not associated } \\
\text { with HIV medication } \\
\text { adherence }\end{array}$ & $\begin{array}{l}\text { Not } \\
\text { included } \\
<13: \beta=- \\
0.13 ; p \\
\text { value }= \\
0.25 \\
13-16: \beta= \\
-0.11 ; p \\
\text { value = } \\
0.26\end{array}$ \\
\hline Muir, $2012^{28}$ & $\begin{array}{l}127 \text { patients } \\
\text { being treated } \\
\text { for glaucoma } \\
\text { at a veteran's } \\
\text { medical center } \\
\text { in Durham, } \\
\text { NC }\end{array}$ & RCT & $\begin{array}{l}\text { Days without } \\
\text { medication } \\
\text { (claims-based) }\end{array}$ & Health literacy & $\begin{array}{l}\text { Comorbid } \\
\text { disability, self- } \\
\text { reported disease } \\
\text { knowledge, and } \\
\text { satisfaction with } \\
\text { care }\end{array}$ & $\begin{array}{l}\text { Days without } \\
\text { medication did not } \\
\text { differ significantly } \\
\text { between literacy } \\
\text { intervention group and } \\
\text { control group those } \\
\text { who did not among } \\
\text { veterans with glaucoma }\end{array}$ & $\begin{array}{l}\text { Not } \\
\text { included } \\
\text { Invention } \\
\text { group }(x= \\
63) \text { vs } \\
\text { control }(x= \\
65),(p= \\
0.71)\end{array}$ \\
\hline $\begin{array}{l}\text { Nwokeji, } \\
2012^{29}\end{array}$ & $\begin{array}{l}166 \text { uninsured } \\
\text { working adults } \\
\text { with major } \\
\text { depression in } \\
\text { demonstration } \\
\text { program, } \\
\text { Texas }\end{array}$ & RCT & $\begin{array}{l}\text { Proportion of } \\
\text { days covered } \\
\text { within } 1 \text { year } \\
\text { (claims-based) }\end{array}$ & Education & $\begin{array}{l}\text { Alcohol diagnosis, } \\
\text { drug diagnosis }\end{array}$ & $\begin{array}{l}\text { Participants with higher } \\
\text { education had a higher } \\
\text { proportion of days } \\
\text { covered by an } \\
\text { antidepressant } \\
\text { prescription compared } \\
\text { to those in a lower } \\
\text { education strata }\end{array}$ & $\begin{array}{l}\text { Not } \\
\text { included }(\beta \\
=0.05 \\
0.02-0.08)\end{array}$ \\
\hline $\begin{array}{l}\text { Parada, } \\
2012^{30}\end{array}$ & $\begin{array}{l}302 \text { Latino } \\
\text { adults with } \\
\text { diabetes in } \\
\text { Southern CA }\end{array}$ & $\begin{array}{l}\text { Secondary } \\
\text { analysis, } \\
\text { RCT }\end{array}$ & $\begin{array}{l}\text { Morisky } \\
\text { medication } \\
\text { adherence } \\
\text { scale (self- } \\
\text { report) }\end{array}$ & Education & $\begin{array}{l}\text { Age, sex, insurance } \\
\text { status, drug } \\
\text { complexity, health } \\
\text { status, patient- } \\
\text { provider relation- } \\
\text { ship }\end{array}$ & $\begin{array}{l}\text { Education was not } \\
\text { associated with } \\
\text { medication adherence } \\
\text { among Latinos with } \\
\text { type } 2 \text { diabetes }\end{array}$ & Included \\
\hline $\begin{array}{l}\text { Quinn, } \\
2016^{31}\end{array}$ & $\begin{array}{l}98 \text { young } \\
\text { black men with } \\
\text { HIV and have } \\
\text { sex with men } \\
\text { in Chicago, IL }\end{array}$ & $\begin{array}{l}\text { Secondary } \\
\text { analysis- } \\
\text { RCT }\end{array}$ & $\begin{array}{l}\text { Percent of the } \\
\text { time they took } \\
\text { medication as } \\
\text { prescribed } \\
\text { (self-report) }\end{array}$ & $\begin{array}{l}\text { Community } \\
\text { violence }\end{array}$ & $\begin{array}{l}\text { Psychological } \\
\text { distress, drug use, } \\
\text { condomless } \\
\text { intercourse }\end{array}$ & $\begin{array}{l}\text { Exposure to high levels } \\
\text { of community violence } \\
\text { was associated with } \\
\text { significantly lower odds } \\
\text { of medication } \\
\text { adherence among black } \\
\text { men with HIV who } \\
\text { have sex with men }\end{array}$ & Included \\
\hline $\begin{array}{l}\text { Sattler, } \\
2014^{32}\end{array}$ & $\begin{array}{l}243 \text { Part D } \\
\text { Medicare } \\
\text { beneficiaries } \\
\text { with type } 2 \\
\text { diabetes in } \\
\text { Georgia }\end{array}$ & $\begin{array}{l}\text { Prospective } \\
\text { cohort study }\end{array}$ & $\begin{array}{l}\text { Proportion of } \\
\text { days covered } \\
\text { (claims-based) }\end{array}$ & $\begin{array}{l}\text { Food and } \\
\text { housing } \\
\text { insecurity }\end{array}$ & & $\begin{array}{l}\text { Food insecurity was not } \\
\text { associated with lower } \\
\text { adherence to oral } \\
\text { hypoglycemic } \\
\text { medications }\end{array}$ & $\begin{array}{l}\text { Not } \\
\text { included } \\
\text { (data from } \\
\text { regression } \\
\text { model not } \\
\text { reported) }\end{array}$ \\
\hline $\begin{array}{l}\text { Shallcross, } \\
2015^{33}\end{array}$ & $\begin{array}{l}55 \text { patients } \\
\text { with epilepsy } \\
\text { in NYC }\end{array}$ & $\begin{array}{l}\text { Cross- } \\
\text { sectional } \\
\text { survey }\end{array}$ & $\begin{array}{l}\text { Morisky } \\
\text { medication } \\
\text { adherence } \\
\text { scale (self- } \\
\text { report) }\end{array}$ & Social support & $\begin{array}{l}\text { Age, sex, income, } \\
\text { seizure frequency, } \\
\text { number of } \\
\text { medications, time } \\
\text { since diagnosis, } \\
\text { and antidepressant } \\
\text { medication usage }\end{array}$ & $\begin{array}{l}\text { Social support was a } \\
\text { significant predictor of } \\
\text { antiepileptic medication } \\
\text { adherence }\end{array}$ & $\begin{array}{l}\text { Not } \\
\text { included }(\beta \\
=.618 ;- \\
.254,- \\
.211)\end{array}$ \\
\hline $\begin{array}{l}\text { Silverman, } \\
2015^{34}\end{array}$ & $\begin{array}{l}287 \text { low- } \\
\text { income adults } \\
\text { with poorly } \\
\text { controlled dia- } \\
\text { betes in Wash- } \\
\text { ington }\end{array}$ & $\begin{array}{l}\text { Secondary } \\
\text { analysis- } \\
\text { RCT }\end{array}$ & $\begin{array}{l}\text { Morisky } \\
\text { medication } \\
\text { adherence } \\
\text { scale (self- } \\
\text { report) }\end{array}$ & $\begin{array}{l}\text { Food } \\
\text { insecurity }\end{array}$ & $\begin{array}{l}\text { Age, gender, } \\
\text { race/ethnicity, } \\
\text { language, } \\
\text { education, marital } \\
\text { status, BMI, } \\
\text { insulin use, } \\
\text { depression, } \\
\text { diabetes distress }\end{array}$ & $\begin{array}{l}\text { Food insecurity was } \\
\text { associated with low } \\
\text { medication adherence } \\
\text { among low-income } \\
\text { adults with poorly con- } \\
\text { trolled diabetes }\end{array}$ & Included \\
\hline
\end{tabular}


Table 2. (continued)

\begin{tabular}{|c|c|c|c|c|c|c|c|}
\hline $\begin{array}{l}\text { First } \\
\text { author, } \\
\text { name }\end{array}$ & Participants & $\begin{array}{l}\text { Study } \\
\text { design }\end{array}$ & $\begin{array}{l}\text { Medication } \\
\text { adherence } \\
\text { measure }\end{array}$ & $\begin{array}{l}\text { Social } \\
\text { determinant/ } \\
\text { s measured }\end{array}$ & Covariates & Main outcome & $\begin{array}{l}\text { Included } \\
\text { in pooled } \\
\text { analysis? }\end{array}$ \\
\hline Soto, $2013^{35}$ & $\begin{array}{l}303 \text { adult men } \\
\text { with HIV who } \\
\text { have sex with } \\
\text { men in } \\
\text { Chicago, IL }\end{array}$ & $\begin{array}{l}\text { Cross- } \\
\text { sectional } \\
\text { survey }\end{array}$ & $\begin{array}{l}\text { Rate ability to } \\
\text { take all } \\
\text { medications as } \\
\text { prescribed } \\
\text { within } 30 \text { days } \\
\text { (self-report) }\end{array}$ & $\begin{array}{l}\text { Crime-related } \\
\text { trauma, } \\
\text { physical } \\
\text { trauma, sexual } \\
\text { trauma, social } \\
\text { support }\end{array}$ & $\begin{array}{l}\text { Disclosure to } \\
\text { family, general } \\
\text { health, treatment } \\
\text { concerns, HIV } \\
\text { stigma }\end{array}$ & $\begin{array}{l}\text { Crime-related trauma, } \\
\text { physical trauma, and } \\
\text { sexual trauma were not } \\
\text { correlated with HIV } \\
\text { MA. Social support was } \\
\text { not correlated with HIV } \\
\text { MA }\end{array}$ & 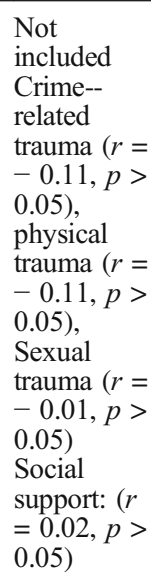 \\
\hline $\begin{array}{l}\text { Surratt, } \\
2015^{36}\end{array}$ & $\begin{array}{l}503 \text { HIV- } \\
\text { positive indi- } \\
\text { viduals in } \\
\text { high-poverty } \\
\text { areas in Miami }\end{array}$ & $\begin{array}{l}\text { Cross- } \\
\text { sectional }\end{array}$ & Self-report & $\begin{array}{l}\text { Food and } \\
\text { housing } \\
\text { insecurity }\end{array}$ & $\begin{array}{l}\text { Depression, } \\
\text { anxiety, traumatic } \\
\text { stress, and } \\
\text { substance } \\
\text { dependence }\end{array}$ & $\begin{array}{l}\text { Food and housing } \\
\text { insecure patients more } \\
\text { likely to report ARV } \\
\text { nonadherence }\end{array}$ & Included \\
\hline $\begin{array}{l}\text { Thames, } \\
2012^{37}\end{array}$ & $\begin{array}{l}181 \text { adults with } \\
\text { HIV and drug } \\
\text { abuse in Los } \\
\text { Angeles, CA }\end{array}$ & $\begin{array}{l}\text { Prospective } \\
\text { cohort study }\end{array}$ & $\begin{array}{l}\text { Percent of } \\
\text { adherence in } \\
\text { past } 6 \text { months } \\
\text { (electronic pill } \\
\text { cap system) }\end{array}$ & $\begin{array}{l}\text { Social } \\
\text { support, } \\
\text { reading } \\
\text { ability/literacy }\end{array}$ & $\begin{array}{l}\text { Age, race, } \\
\text { Depression, drug } \\
\text { use, provider } \\
\text { satisfaction }\end{array}$ & $\begin{array}{l}\text { Reading ability/literacy } \\
\text { was not associated with } \\
\text { HIV medication adher- } \\
\text { ence among African } \\
\text { Americans or Cauca- } \\
\text { sians }\end{array}$ & $\begin{array}{l}\text { Not } \\
\text { included } \\
\text { African } \\
\text { Americans } \\
(p=0.56) \\
\text { Caucasians } \\
(p=21)\end{array}$ \\
\hline $\begin{array}{l}\text { Trimble, } \\
2013^{38}\end{array}$ & $\begin{array}{l}272 \text { women } \\
\text { with HIV } \\
\text { receiving care } \\
\text { at public clinic } \\
\text { in Southern } \\
\text { Texas }\end{array}$ & $\begin{array}{l}\text { Cross- } \\
\text { sectional } \\
\text { survey }\end{array}$ & $\begin{array}{l}\text { Morisky } \\
\text { medication } \\
\text { adherence } \\
\text { scale (self- } \\
\text { report) }\end{array}$ & $\begin{array}{l}\text { Intimate } \\
\text { partner } \\
\text { violence }\end{array}$ & $\begin{array}{l}\text { Age, race, } \\
\text { education, } \\
\text { employment, living } \\
\text { with partner }\end{array}$ & $\begin{array}{l}\text { Women who } \\
\text { experienced intimate } \\
\text { partner violence in past } \\
12 \text { months had lower } \\
\text { HIV MA scores on } \\
\text { average than women } \\
\text { who did not }\end{array}$ & $\begin{array}{l}\text { Not } \\
\text { included }(x \\
=5.49) \text { vs } \\
(x=6.57) \\
(p<0.001)\end{array}$ \\
\hline $\begin{array}{l}\text { Vissman, } \\
2013^{39}\end{array}$ & $\begin{array}{l}66 \text { Latino } \\
\text { patients with } \\
\text { HIV in Wake } \\
\text { Forest, NC }\end{array}$ & $\begin{array}{l}\text { Cross- } \\
\text { sectional } \\
\text { survey }\end{array}$ & $\begin{array}{l}2 \text { questions } \\
\text { about taking } \\
\text { HIV } \\
\text { medications } \\
\text { within past } 30 \\
\text { days (self- } \\
\text { report) }\end{array}$ & $\begin{array}{l}\text { Employment, } \\
\text { social support }\end{array}$ & $\begin{array}{l}\text { Perceived } \\
\text { behavioral control, } \\
\text { education }\end{array}$ & $\begin{array}{l}\text { Employment was not } \\
\text { associated with HIV } \\
\text { medication adherence. } \\
\text { Social support was not } \\
\text { associated with HIV } \\
\text { MA }\end{array}$ & Included \\
\hline $\begin{array}{l}\text { Voisin, } \\
2016^{40}\end{array}$ & $\begin{array}{l}92 \text { young } \\
\text { black men who } \\
\text { have sex with } \\
\text { men and are } \\
\text { HIV positive } \\
\text { in Chicago, IL }\end{array}$ & $\begin{array}{l}\text { Secondary } \\
\text { analysis- } \\
\text { RCT }\end{array}$ & $\begin{array}{l}\text { Percentage of } \\
\text { the time they } \\
\text { took } \\
\text { medication as } \\
\text { prescribed } \\
\text { (self-report) }\end{array}$ & Social support & $\begin{array}{l}\text { Self-efficacy, social } \\
\text { stigma, family } \\
\text { acceptance, } \\
\text { marijuana use, } \\
\text { alcohol use }\end{array}$ & $\begin{array}{l}\text { Social support was not } \\
\text { associated with HIV } \\
\text { MA among young } \\
\text { black men who have } \\
\text { HIV and have sex with } \\
\text { other men }\end{array}$ & Included \\
\hline $\begin{array}{l}\text { Weiser, } \\
2013^{41}\end{array}$ & $\begin{array}{l}188 \text { HIV+ } \\
\text { adults taking } \\
\text { ART and } \\
\text { scoring below } \\
\text { 90\% correct on } \\
\text { TOFHLA }\end{array}$ & Cohort & Self-report & $\begin{array}{l}\text { Food and } \\
\text { housing } \\
\text { insecurity }\end{array}$ & Substance abuse & $\begin{array}{l}\text { Food and housing } \\
\text { insecurity increased } \\
\text { odds of nonadherence, } \\
\text { inadequate viral } \\
\text { suppression, and low } \\
\text { CD4 counts }\end{array}$ & Included \\
\hline $\begin{array}{l}\text { Welty, } \\
2010^{42}\end{array}$ & $\begin{array}{l}143 \text { adults with } \\
\text { epilepsy who } \\
\text { were members } \\
\text { of Epilepsy. } \\
\text { com website } \\
\text { (United States) }\end{array}$ & $\begin{array}{l}\text { Cross- } \\
\text { sectional } \\
\text { survey }\end{array}$ & $\begin{array}{l}\text { Several } \\
\text { questions on } \\
\text { transportation } \\
\text { limiting MA } \\
\text { (self-report) }\end{array}$ & Transportation & $\mathrm{N} / \mathrm{a}$ & $\begin{array}{l}\text { Not driving a car was } \\
\text { associated with } \\
\text { reporting difficulty } \\
\text { picking up prescriptions } \\
\text { for adults with epilepsy }\end{array}$ & Included \\
\hline $\begin{array}{l}\text { Wisnivesky, } \\
2012^{43}\end{array}$ & $\begin{array}{l}87 \text { elderly } \\
\text { Hispanic } \\
\text { patients with } \\
\text { asthma in } \\
\text { NYC and } \\
\text { Chicago }\end{array}$ & $\begin{array}{l}\text { Prospective } \\
\text { cohort study }\end{array}$ & $\begin{array}{l}\text { Medication } \\
\text { Adherence } \\
\text { Reporting } \\
\text { Scale (MARS) } \\
\text { (self-report) }\end{array}$ & $\begin{array}{l}\text { Language/ } \\
\text { literacy }\end{array}$ & $\begin{array}{l}\text { Sociodemographic } \\
\text { characteristics, } \\
\text { asthma history, } \\
\text { comorbid } \\
\text { conditions, } \\
\text { depression, health } \\
\text { literacy }\end{array}$ & $\begin{array}{l}\text { Hispanic patients with } \\
\text { limited English } \\
\text { proficiency were less } \\
\text { likely than non- } \\
\text { Hispanic patients to be } \\
\text { adherent to asthma } \\
\text { medications }\end{array}$ & $\begin{array}{l}\text { Not } \\
\text { included } \\
\text { (odds ratio } \\
\text { only } \\
\text { reported } \\
\text { adherence } \\
\text { by race) }\end{array}$ \\
\hline $\begin{array}{l}\text { Zullig, } \\
2013^{44}\end{array}$ & $\begin{array}{l}406 \text { adults who } \\
\text { had }\end{array}$ & & $\begin{array}{l}\text { Morisky } \\
\text { medication }\end{array}$ & $\begin{array}{l}\text { Financial } \\
\text { status, }\end{array}$ & $\begin{array}{l}\text { Life chaos, } \\
\text { demographics, }\end{array}$ & $\begin{array}{l}\text { Financial status, } \\
\text { employment, education, }\end{array}$ & Included \\
\hline
\end{tabular}


Table 2. (continued)

\begin{tabular}{|c|c|c|c|c|c|c|c|}
\hline $\begin{array}{l}\text { First } \\
\text { author, } \\
\text { name }\end{array}$ & Participants & $\begin{array}{l}\text { Study } \\
\text { design }\end{array}$ & $\begin{array}{l}\text { Medication } \\
\text { adherence } \\
\text { measure }\end{array}$ & $\begin{array}{l}\text { Social } \\
\text { determinant/ } \\
\text { s measured }\end{array}$ & Covariates & Main outcome & $\begin{array}{l}\text { Included } \\
\text { in pooled } \\
\text { analysis? }\end{array}$ \\
\hline & $\begin{array}{l}\text { hypertension } \\
\text { and myocardial } \\
\text { infarction }<3 \\
\text { years }\end{array}$ & $\begin{array}{l}\text { Secondary } \\
\text { analysis- } \\
\text { RCT }\end{array}$ & $\begin{array}{l}\text { adherence } \\
\text { scale (self- } \\
\text { report) }\end{array}$ & $\begin{array}{l}\text { employment, } \\
\text { education, } \\
\text { health literacy }\end{array}$ & $\begin{array}{l}\text { including sex, race, } \\
\text { marital status }\end{array}$ & $\begin{array}{l}\text { and health literacy were } \\
\text { not associated with } \\
\text { medication } \\
\text { nonadherence }\end{array}$ & \\
\hline $\begin{array}{l}\text { Zullig, } \\
2014^{45}\end{array}$ & $\begin{array}{l}23 \text { patients } \\
\text { with } \\
\text { cardiovascular } \\
\text { risk factor } \\
\text { receiving } \\
\text { primary care at } \\
\text { veteran's } \\
\text { medical center } \\
\text { in Durham, } \\
\text { NC }\end{array}$ & $\begin{array}{l}\text { Prospective } \\
\text { cohort study }\end{array}$ & $\begin{array}{l}\text { Morisky } \\
\text { medication } \\
\text { adherence } \\
\text { scale (self- } \\
\text { report) and } \\
\text { medication } \\
\text { possession ra- } \\
\text { tio (claims- } \\
\text { based) }\end{array}$ & Health literacy & $\mathrm{N} / \mathrm{a}$ & $\begin{array}{l}\text { Health literacy } \\
\text { intervention improved } \\
\text { self-reported medica- } \\
\text { tion adherence but not } \\
\text { medication possession } \\
\text { ratio }\end{array}$ & $\begin{array}{l}\text { Not } \\
\text { included } \\
p=0.73\end{array}$ \\
\hline
\end{tabular}

The 29 studies consisted of 10 cross-sectional surveys, eight prospective cohort designs, one randomized controlled trial (RCT), and the secondary analysis of data from eight RCTs and two prospective cohort studies (see Table 2). All but one study focused on a disease-specific population; the most common chronic conditions were HIV $(n=9)$, diabetes $(n=6)$, and cardiovascular disease $(n=4)$. Sample sizes ranged from 23 to 3401. Eleven of the 29 studies were based on less than 200 participants. More than half $(n=16)$ of the studies were restricted to subjects with a specific characteristic such as Hispanic ethnicity $(n=3)$, veteran $(n=3)$, male $(n=3)$, or low income $(n=3)$.
Of the 29 included studies, 12 studies examined an economic stability-related SDH factor, 10 studies included an education-related SDH, 10 studies included a social and community context-related SDH, eight studies consisted of SDH related to health and health care, and four studies examined SDH related to the neighborhood and the built environment. More than half of the included studies $(n=16)$ only evaluated one SDH. Table 2 summarizes each of the studies included in the systematic review and their outcome.

Figures 2 and 3 display the pooled adjusted and unadjusted odds ratio for each SDH subdomain and overall. In both pooled analyses, food insecurity and housing instability

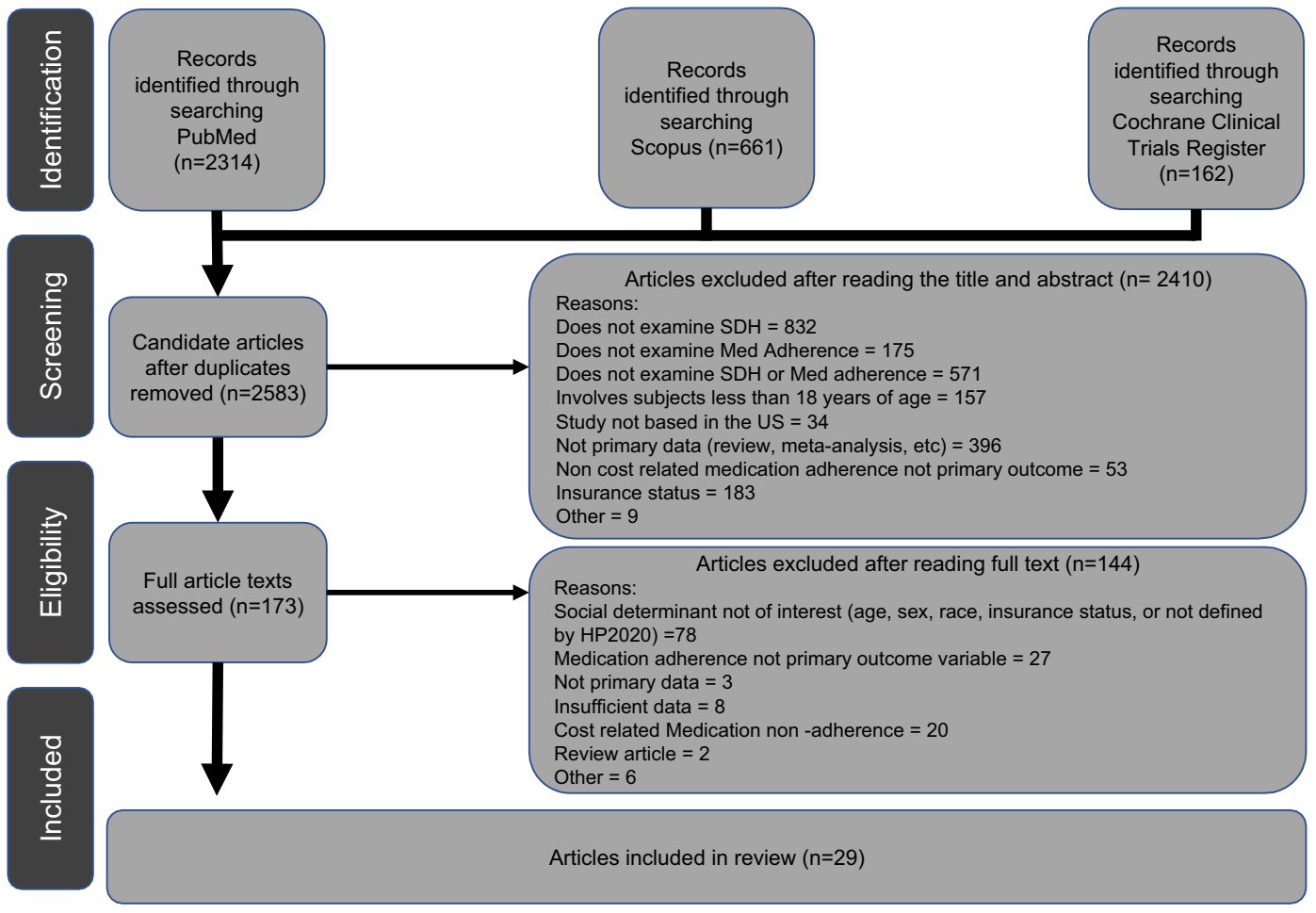

Figure 1 PRISMA flow diagram. 


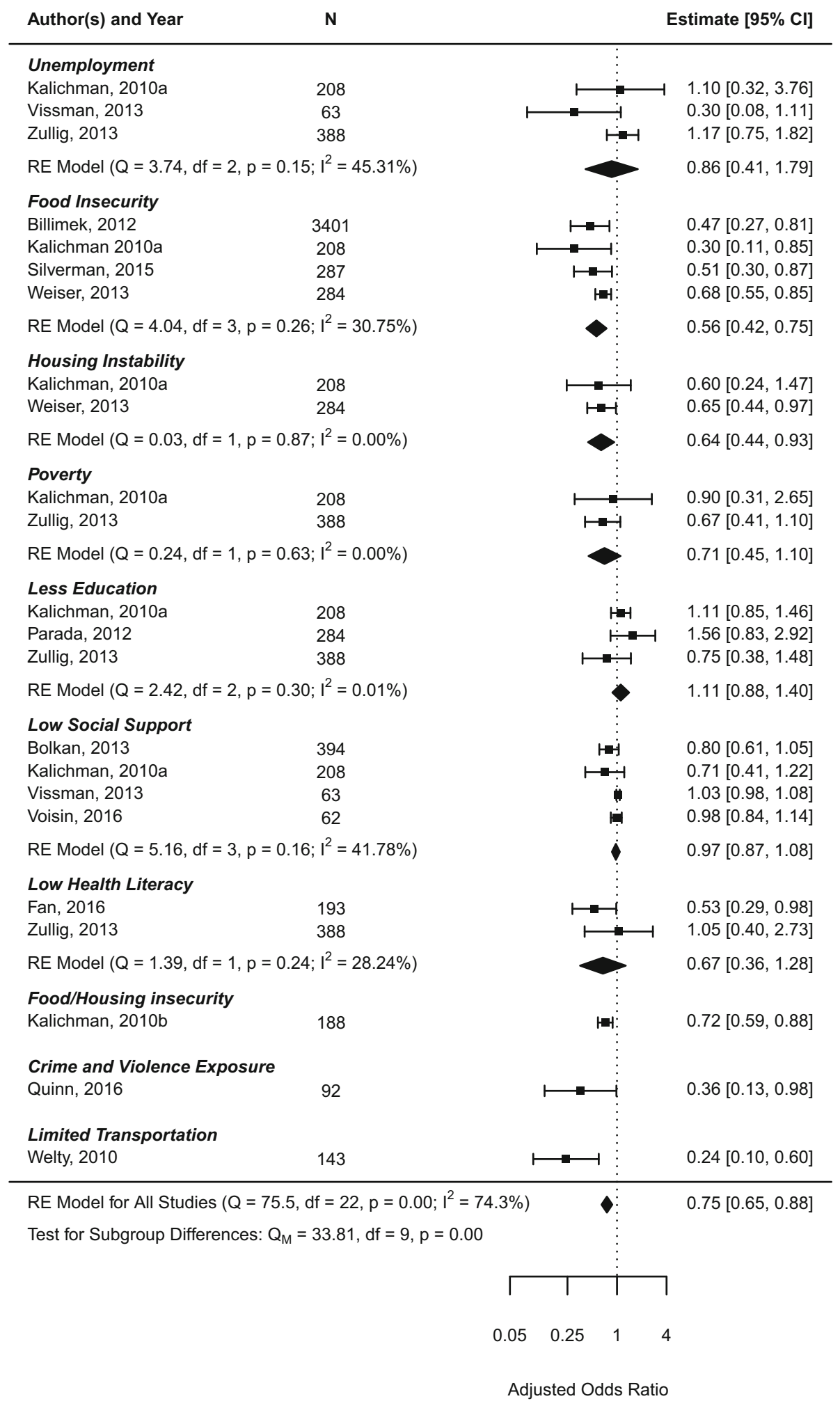

Figure 2 Pooled adjusted odds ratios.

demonstrated a consistent and statistically significant association with medication adherence (Figs. 2 and 3). Food insecurity demonstrated the most significant impact, with a pooled aOR of $0.56(95 \%$ CI $0.42-0.7)$ and an OR of 0.58 (95\% CI $0.43-0.78)$. Housing instability was also significantly associated with MA in our pooled analysis with an aOR of 0.64 (95\% CI 0.44-0.93). Unemployment, poverty, and inadequate social support were not associated with MA. Lower health literacy and education were significantly associated with adherence in the pooled unadjusted 


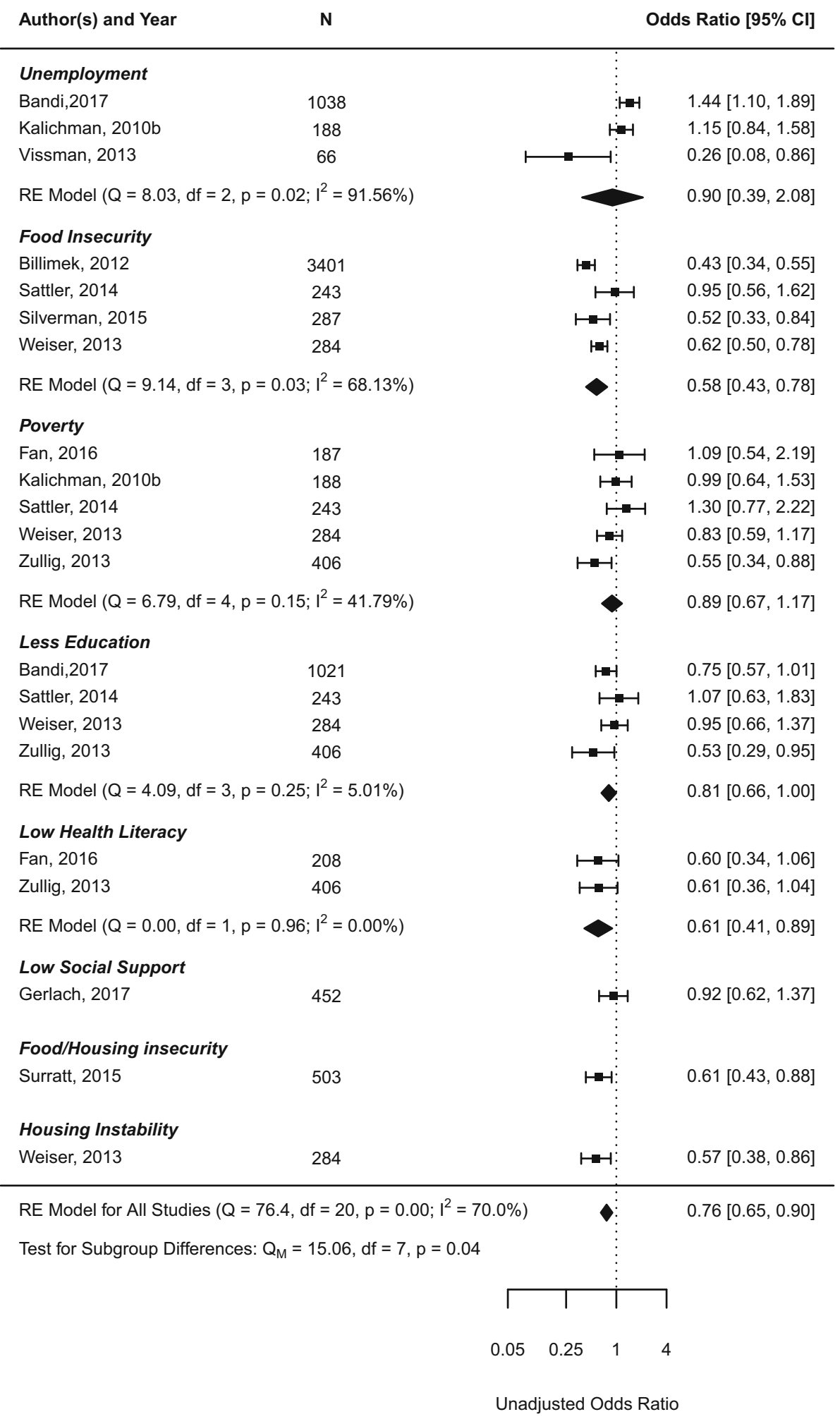

Figure 3 Pooled unadjusted odds ratios.

analysis only. When we pooled the SDH factors across all of the domains, we found that adverse SDH were significantly associated with $\mathrm{MA}$ in both the pooled adjusted (aOR = $0.75 ; 95 \%$ CI $0.65-0.88)$ and unadjusted $(\mathrm{OR}=0.76$; $95 \%$ CI 0.65-0.90) meta-analyses.

\section{DISCUSSION}

This systematic review of the evidence of the influence of social determinants of health on medication adherence revealed relatively few studies on this topic. Medication adherence was most commonly studied in the HP2020 economic 
stability domain. Some subdomains of SDH have received no attention in the literature such as the impact of incarceration or the quality of housing on MA. When we estimated the relationship between SDH and medication adherence overall, we found that adverse social determinants were associated with lower adherence. For specific SDH factors, only food insecurity and housing instability were correlated with poor medication adherence. For all other specific social determinants, the evidence was inconsistent or there was only one study that evaluated the specific determinant.

Food insecurity was associated with poorer medication adherence in this study based on study samples that included patients with diabetes or HIV. These findings are consistent with two systematic review studies that evaluated the influence of food insecurity on adherence. ${ }^{7,12}$ There are multiple reasons why food insecurity negatively impacts medication nonadherence. Lack of food increases hunger and results in people prioritizing basic survival needs over other competing issues such as medication adherence. ${ }^{46}$ Food insecurity is also associated with decreased self-efficacy which can adversely affect mental health and adherence. ${ }^{46}$ Finally, patients can experience more side effects when their medications are not taken with food and/or their nutritional status is compromised. ${ }^{7}$

Housing instability was also associated with poorer medication adherence. Three studies examined housing instability primarily and two other studies examined a combination of housing instability and food insecurity. Similar to food insecurity, when people are unstably housed, they are not meeting their basic needs. When basic needs compete with health problems, people may prioritize getting their basic needs met over their health, thus explaining why housing instability is associated with lower medication adherence.

Despite the heterogeneity of included studies in terms of study design, measurement of SDH and medication adherence, and statistical analyses, the overall pooled results revealed a significant association between $\mathrm{SDH}$ and medication adherence. These findings suggest that this relationship warrants more attention and research by health care providers and policymakers. The vast majority of interventions aimed at improving medication adherence focus on patient education, medication regimen management, and more frequent monitoring including reminders. ${ }^{47,48}$ If we want to improve medication adherence, we must appreciate the broader context in which our patients live and design interventions and policies that also address adverse social determinants that make it hard for people to adhere. ${ }^{49}$

Structural determinants of health inequities, such as employment, income, and education, were not significantly associated with medication adherence. Structural determinants impact one's socioeconomic position in society which influences intermediary determinants of health that directly influence health outcomes. Intermediary determinants of health include material circumstances, psychosocial circumstances, behavioral and/or biological factors, and the health system. The structural determinants of health inequities impact health outcomes indirectly through the intermediary determinants. When both structural and intermediary determinants of health are included simultaneously in statistical models, it likely results in over adjustment. Models that include both structural and intermediary SDH factors should conduct mediation analysis to determine the extent to which the influence of structural determinants is explained by intermediary determinants. It is possible that poverty, education, and employment were not significantly associated with MA because of the presence of other intermediary SDH factors in the analyses. ${ }^{50-53}$

There was significant variation in how the SDH factor and MA were measured in included studies, which also likely influenced study results. For example, even though the majority of studies that measured poverty included a measure of income, several studies measured personal income, several measured household income, and one study measured household income adjusted for family size. Among the 10 studies that examined the relationship between social support and MA, only two used the same social support scale. ${ }^{23,26}$ Furthermore, not all of the social support scales measured the same dimensions of social support. Some studies chose social support scales that measured emotional and instrumental social support, ${ }^{20,23,26,39}$ some selected measures of general social support, ${ }^{22,33,35,54}$ and others selected measures of social support specific to medication adherence. ${ }^{37,40}$

Similarly, the studies measured MA using a variety of methods and this could also have contributed to the inconsistency of result findings across the studies. The most common method of adherence was self-report $(n=$ 24); relatively few studies used an objective MA measure such as prescription claims or electronic pill cap monitoring. Of the 24 studies that used a self-reported adherence measure, only 11 used a validated scale such as the Morisky Medication Adherence Scale. There was also significant variation in the time frame used to measure medication adherence. Across the 29 studies, the MA time frame ranged between 1 week and 1 year. Additionally, MA was often measured as a continuous variable, and later dichotomized using a threshold value that ranged between 70 and $90 \%$. A small number of the studies treated MA as a continuous variable in regression analyses.

Many of the studies had methodological limitations that likely compromised study findings and therefore resulted in inconsistent findings across the SDH domains. One-third of the studies included in this review were cross-sectional studies that measured SDH and MA at one point in time, even though both can vary over time. Some of the studies had inadequate power to find a significant difference either because of a small sample size $33,39,40,45$ or because their study sample did not include sufficient variation in a SDH factor. ${ }^{20,21,30,32,35,37,44}$ Less than half of the studies evaluated the influence of two or more SDH factors on MA. In addition, relatively few studies accounted for other types of factors that may influence MA beside demographic characteristics such as age, sex, and race. 
Rarely did studies take into account the complexity of the medication regimen, the severity of the disease or presence of comorbidities, or the quality of the patient-provider relationship.

The results of this systematic review and metaanalysis must be interpreted in the context of the following limitations. First, this search was restricted to papers written in English and studies conducted in the US which limits the generalizability of the findings of this systematic review. As stated previously, significant differences in health care systems and social spending per capita by other developed nations limit the ability to make comparisons. Second, we excluded health insurance coverage because there is already ample evidence of its negative impact on MA. Third, we did not evaluate the relationship between SDH and medication adherence by medical condition; it is possible that the relationship varies by medical condition. Finally, we relied on the HP2020 as our SDH framework. Currently, there is no universal SDH model that has been widely adopted. If we had selected a different framework, it would have resulted in a different set of SDH factors included in the review. For example, the Institute of Medicine SDH model ${ }^{55}$ considers stress and mental health as SDH factors; HP2020 does not.

In conclusion, despite the importance of medication adherence in achieving good health outcomes, particularly for people with chronic illnesses, this systematic review identified relatively few studies that have examined the relationship between social determinants of health and medication adherence. The overall results demonstrated that social adversities are associated with lower adherence. A similar result was also revealed for food insecurity and housing instability. Wide variation in how SDH factors and MA were measured as well as methodological limitations of the studies likely contributed to inconsistent results across the other SDH domains. This systematic review and meta-analysis underscores the critical need for more rigorous research in this area.

Corresponding Author: Marcee E. Wilder, M.D., M.P.H.; Department of Emergency Medicine, George Washington University, Medical Faculty Associates, Washington, DC, USA (e-mail: M.wilder@mfa. gwu.edu).

Funding: This project was supported by the National Institute on Minority Health and Health Disparities (NIMHD) grant no. R01MD011607.

\section{Compliance with Ethical Standards:}

Conflict of Interest: The authors declare that they do not have a conflict of interest.

Disclaimer: This content is solely the responsibility of the authors and does not necessarily represent the official views of the NIMHD or the National Institutes of Health.

\section{REFERENCES}

1. Haynes RB, McDonald HP, Garg AX. Helping patients follow prescribed treatment: clinical applications. JAMA. 2002;288(22):2880-3. https:// doi.org/10.1001/jama.288.22.2880.

2. DiMatteo MR, Giordani PJ, Lepper HS, Croghan TW. Patient adherence and medical treatment outcomes: a meta-analysis. Med Care. 2002;40(9):794811. https://doi.org/10.1097/00005650-200209000-00009.

3. World Health Organization., Sabaté E. Adherence to long-term therapies : evidence for action. World Health Organization; 2003:xv, 198 p.

4. Agarwal R, Gupta A, Fendrick AM. Value-Based Insurance Design Improves Medication Adherence Without An Increase In Total Health Care Spending. Health Aff (Millwood). 2018;37(7):1057-1064. https:// doi.org/10.1377/hlthaff.2017.1633.

5. Mishuk AU, Fasina I, Qian J. Impact of U.S. federal and state generic drug policies on drug use, spending, and patient outcomes - A systematic review. Res Social Adm Pharm. 2019; https://doi.org/10.1016/j.sapharm.2019.08.031.

6. Zhang JX, Bhaumik D, Huang ES, Meltzer Do. Change in Insurance Status and Cost-related Medication Non-adherence among Older U.S. Adults with Diabetes from 2010 to 2014. J Health Med Econ. 2018;4(2) https://doi.org/10.21767/2471-9927.100040

7. Walker RJ, Smalls BL, Campbell JA, Strom Williams JL, Egede LE. Impact of social determinants of health on outcomes for type 2 diabetes: a systematic review. Endocrine. 2014;47(1):29-48. https://doi.org/10. 1007/s12020-014-0195-0.

8. Aidala AA, Wilson MG, Shubert V, et al. Housing Status, Medical Care, and Health Outcomes Among People Living With HIV/AIDS: A Systematic Review. Am J Public Health. 2016;106(1):e1-e23. https://doi.org/10. 2105/AJPH.2015.302905.

9. Chop E, Duggaraju A, Malley A, et al. Food insecurity, sexual risk behavior, and adherence to antiretroviral therapy among women living with HIV: A systematic review. Health Care Women Int. 2017;38(9):927944. https://doi.org/10.1080/07399332.2017.1337774.

10. Geter A, Sutton MY, Hubbard McCree D. Social and structural determinants of HIV treatment and care among black women living with HIV infection: a systematic review: 2005-2016. AIDS Care. 2018;30(4):409416. https://doi.org/10.1080/09540121.2018.1426827.

11. Oosterom-Calo R, van Ballegooijen AJ, Terwee CB, et al. Determinants of adherence to heart failure medication: a systematic literature review. Heart Fail Rev. 2013;18(4):409-27. https://doi.org/10.1007/s10741-012-9321-3.

12. Singer AW, Weiser SD, McCoy SI. Does Food Insecurity Undermine Adherence to Antiretroviral Therapy? A Systematic Review. AIDS Behav. 2015;19(8):1510-26. https://doi.org/10.1007/s10461-014-0873-1.

13. Liberati A, Altman DG, Tetzlaff J, et al. The PRISMA statement for reporting systematic reviews and meta-analyses of studies that evaluate health care interventions: explanation and elaboration. PLoS Med. 2009;6(7):e1000100. https://doi.org/10.1371/journal.pmed. 1000100.

14. Wilder ME, McCarthy M, Jensen C, Kulie P, Levett P. A systematic review of social determinants of health and their impact on medication adherence. https://www.crd.york.ac.uk/prospero/display_record.php? ID = CRD42019119105. Accessed 8 Sep 2019.

15. Services USDoHaH. Healthy People 2020. Accessed September 8, 2019, https://www.healthypeople.gov/2020/topics-objectives/topic/social-determinants-of-health.

16. Bradley EH, Elkins BR, Herrin J, Elbel B. Health and social services expenditures: associations with health outcomes. BMJ Qual Saf. 2011;20(10):826-31. https://doi.org/10.1136/bmjqs.2010.048363.

17. Bandi P, Goldmann E, Parikh NS, Farsi P, Boden-Albala B. Age-Related Differences in Antihypertensive Medication Adherence in Hispanics: A Cross-Sectional Community-Based Survey in New York City, 2011-2012. Prev Chronic Dis. 2017;14:E57. https://doi.org/10.5888/pcd14.160512.

18. Bauer AM, Schillinger D, Parker MM, et al. Health literacy and antidepressant medication adherence among adults with diabetes: the diabetes study of Northern California (DISTANCE). J Gen Intern Med. 2013;28(9):1181-7. https://doi.org/10.1007/s11606-013-2402-8.

19. Billimek J, Sorkin DH. Food insecurity, processes of care, and selfreported medication underuse in patients with type 2 diabetes: results from the California Health Interview Survey. Health Serv Res. 2012;47(6):215968. https://doi.org/10.1111/j.1475-6773.2012.01463.x.

20. Bolkan CR, Bonner LM, Campbell DG, et al. Family involvement, medication adherence, and depression outcomes among patients in veterans affairs primary care. Psychiatr Serv. 2013;64(5):472-8. https:// doi.org/10.1176/appi.ps.201200160.

21. Fan JH, Lyons SA, Goodman MS, Blanchard MS, Kaphingst KA. Relationship Between Health Literacy and Unintentional and Intentional Medication Nonadherence in Medically Underserved Patients With Type 2 
Diabetes. Diabetes Educ. 2016;42(2):199-208. https://doi.org/10.1177/ 0145721715624969.

22. Gerlach LB, Kavanagh J, Watkins D, Chiang C, Kim HM, Kales HC. With a little help from my friends?: racial and gender differences in the role of social support in later-life depression medication adherence. Int Psychogeriatr. 2017;29(9):1485-1493. https://doi.org/10.1017/ S104161021700076X

23. Johnson VR, Jacobson KL, Gazmararian JA, Blake SC. Does social support help limited-literacy patients with medication adherence? A mixed methods study of patients in the Pharmacy Intervention for Limited Literacy (PILL) study. Patient Educ Couns. 2010;79(1):14-24. https://doi.org/10.1016/j.pec.2009.07.002.

24. Kalichman SC, Cherry C, Amaral C, et al. Health and treatment implications of food insufficiency among people living with HIV/AIDS, Atlanta, Georgia. J Urban Health. 2010;87(4):631-41. https://doi.org/ 10.1007/s11524-010-9446-4.

25. Kalichman SC, Hernandez D, Cherry C, Kalichman MO, Washington C, Grebler T. Food insecurity and other poverty indicators among people living with HIV/AIDS: effects on treatment and health outcomes. J Community Health. 2014;39(6):1133-9. https://doi.org/10.1007/ s10900-014-9868-0.

26. Kripalani S, Goggins $\mathbf{K}$, Nwosu S, et al. Medication Nonadherence Before Hospitalization for Acute Cardiac Events. J Health Commun. 2015;20 Suppl 2:34-42. https://doi.org/10.1080/10810730.2015. 1080331 .

27. Markowitz SM, O'Cleirigh C, Hendriksen ES, Bullis JR, Stein M, Safren SA. Childhood sexual abuse and health risk behaviors in patients with HIV and a history of injection drug use. AIDS Behav. 2011;15(7):1554-60. https://doi.org/10.1007/s10461-010-9857-y.

28. Muir KW, Ventura A, Stinnett SS, Enfiedjian A, Allingham RR, Lee PP. The influence of health literacy level on an educational intervention to improve glaucoma medication adherence. Patient Educ Couns. 2012;87(2):160-4. https://doi.org/10.1016/j.pec.2011.09.009.

29. Nwokeji ED, Bohman TM, Wallisch L, et al. Evaluating patient adherence to antidepressant therapy among uninsured working adults diagnosed with major depression: results of the Texas Demonstration to Maintain Independence and Employment study. Adm Policy Ment Health. 2012;39(5):374-82. https://doi.org/10.1007/s10488-011-0354-z.

30. Parada H, Horton LA, Cherrington A, Ibarra L, Ayala GX. Correlates of medication nonadherence among Latinos with type 2 diabetes. Diabetes Educ. 2012;38(4):552-61. https://doi.org/10.1177/ 0145721712445215.

31. Quinn K, Voisin DR, Bouris A, Schneider J. Psychological distress, drug use, sexual risks and medication adherence among young HIV-positive Black men who have sex with men: exposure to community violence matters. AIDS Care. 2016;28(7):866-72. https://doi.org/10.1080/ 09540121.2016.1153596.

32. Sattler EL, Lee JS, Bhargava V. Food insecurity and medication adherence in low-income older Medicare beneficiaries with type 2 diabetes. J Nutr Gerontol Geriatr. 2014;33(4):401-17. https://doi.org/ 10.1080/21551197.2014.959680.

33. Shallcross AJ, Becker DA, Singh A, et al. Psychosocial factors associated with medication adherence in ethnically and socioeconomically diverse patients with epilepsy. Epilepsy Behav. 2015;46:242-5. https://doi.org/10.1016/j.yebeh.2015.01.034

34. Silverman J, Krieger J, Kiefer M, Hebert P, Robinson J, Nelson K. The Relationship Between Food Insecurity and Depression, Diabetes Distress and Medication Adherence Among Low-Income Patients with PoorlyControlled Diabetes. J Gen Intern Med. 2015;30(10):1476-80. https:// doi.org/10.1007/s11606-015-3351-1.

35. Soto T, Komaie G, Neilands TB, Johnson MO. Exposure to crime and trauma among HIV-infected men who have sex with men: associations with HIV stigma and treatment engagement. J Assoc Nurses AIDS Care. 2013;24(4):299-307. https://doi.org/10.1016/j.jana.2012.11.008.

36. Surratt HL, O'Grady CL, Levi-Minzi MA, Kurtz SP. Medication adherence challenges among HIV positive substance abusers: the role of food and housing insecurity. AIDS Care. 2015;27(3):307-14. https:// doi.org/10.1080/09540121.2014.967656.

37. Thames AD, Moizel J, Panos SE, et al. Differential predictors of medication adherence in HIV: findings from a sample of African American and Caucasian HIV-positive drug-using adults. AIDS Patient Care STDS. 2012;26(10):621-30. https://doi.org/10.1089/apc.2012.0157.

38. Trimble DD, Nava A, McFarlane J. Intimate partner violence and antiretroviral adherence among women receiving care in an urban Southeastern Texas HIV clinic. J Assoc Nurses AIDS Care. 2013;24(4):331-40. https://doi.org/10.1016/j.jana.2013.02.006.
39. Vissman AT, Young AM, Wilkin AM, Rhodes SD. Correlates of HAART adherence among immigrant Latinos in the Southeastern United States. AIDS Care. 2013;25(3):356-63. https://doi.org/10.1080/09540121. 2012.701722 .

40. Voisin DR, Guinn K, Kim DH, Schneider J. A Longitudinal Analysis of Antiretroviral Adherence Among Young Black Men Who Have Sex With Men. J Adolesc Health. 2017;60(4):411-416. https://doi.org/10.1016/j. jadohealth.2016.10.428.

41. Weiser SD, Yuan C, Guzman D, et al. Food insecurity and HIV clinical outcomes in a longitudinal study of urban homeless and marginally housed HIV-infected individuals. AIDS. 2013;27(18):2953-8. https://doi. org/10.1097/01.aids.0000432538.70088.a3.

42. Welty TE, Willis SL, Welty EA. Effect of limited transportation on medication adherence in patients with epilepsy. J Am Pharm Assoc (2003). 2010;50(6):698-703. https://doi.org/10.1331/JAPhA.2010. 09081 .

43. Wisnivesky JP, Krauskopf $\mathbf{K}$, Wolf $\mathbf{M S}$, et al. The association between language proficiency and outcomes of elderly patients with asthma. Ann Allergy Asthma Immunol. 2012;109(3):179-84. https://doi.org/10.1016/ j.anai.2012.06.016

44. Zullig LL, Shaw RJ, Crowley MJ, et al. Association between perceived life chaos and medication adherence in a postmyocardial infarction population. Circ Cardiovasc Qual Outcomes. 2013;6(6):619-25. https:// doi.org/10.1161/CIRCOUTCOMES.113.000435.

45. Zullig LL, McCant F, Melnyk SD, Danus S, Bosworth HB. A health literacy pilot intervention to improve medication adherence using Meducation ${ }^{\circledR}$ technology. Patient Educ Couns. 2014;95(2):288-91. https://doi.org/10.1016/j.pec.2014.02.004.

46. Young S, Wheeler AC, McCoy SI, Weiser SD. A review of the role of food insecurity in adherence to care and treatment among adult and pediatric populations living with HIV and AIDS. AIDS Behav. 2014;18 Suppl 5:S505-15. https://doi.org/10.1007/s10461-013-0547-4.

47. Wilhelmsen NC, Eriksson T. Medication adherence interventions and outcomes: an overview of systematic reviews. Eur J Hosp Pharm. 2019;26(4): 187-192. https://doi.org/10.1136/ejhpharm-2018-001725.

48. Kini V, Ho PM. Interventions to Improve Medication Adherence: A Review. JAMA. 2018;320(23):2461-2473. https://doi.org/10.1001/ jama.2018.19271.

49. Marmot M. The health gap: Doctors and the social determinants of health. Scand J Public Health. 2017;45(7):686-693. https://doi.org/10. $1177 / 1403494817717448$.

50. Solar O, Irwin A. A conceptual framework for action on the social determinants of health. Social Determinants of Health Discussion Paper 2 DEBATES, POLICY \& PRACTICE, CASE STUDIES: World Health Organization; 2010.

51. Bijlsma MJ, Tarkiainen L, Myrskylä M, Martikainen P. Unemployment and subsequent depression: A mediation analysis using the parametric G-formula. Soc Sci Med. 2017;194:142-150. https://doi.org/10.1016/j. socscimed.2017.10.011.

52. Houle J, Lauzier-Jobin F, Beaulieu MD, et al. Socioeconomic status and glycemic control in adult patients with type 2 diabetes: a mediation analysis. BMJ Open Diabetes Res Care. 2016;4(1):e000184. https://doi. org/10.1136/bmjdrc-2015-000184.

53. Kalichman SC, Kalichman MO. HIV-Related Stress and Life Chaos Mediate the Association Between Poverty and Medication Adherence Among People Living with HIV/AIDS. J Clin Psychol Med Settings. 2016;23(4):420-430. https://doi.org/10.1007/s10880-016-9481-8.

54. Kalichman SC, Grebler T. Stress and poverty predictors of treatment adherence among people with low-literacy living with HIV/AIDS. Psychosom Med. 2010;72(8):810-6. https://doi.org/10.1097/PSY. Ob013e3181f01be3.

55. National Academies of Sciences Engineering and Medicine (U.S.). Committee on Educating Health Professionals to Address the Social Determinants of Health, Institute of Medicine (U.S.), Institute of Medicine (U.S.). Global Forum on Innovation in Health Professional Education. A framework for educating health professionals to address the social determinants of health. National Academies Press,; 2016:1 online resource (1 PDF file (xvii, 154 pages)). Accessed December 12, 2016. https://www.ncbi.nlm.nih.gov/books/NBK395983/.

Publisher's Note: Springer Nature remains neutral with regard to jurisdictional claims in published maps and institutional affiliations. 